\title{
Erratum
}

\section{An upper limit of gaseous water abundance in Chamaeleon-MMS1 as observed with ODIN}

\author{
A. Klotz ${ }^{1}$, J. Harju ${ }^{2}$, I. Ristorcelli ${ }^{1}$, M. Juvela ${ }^{2}$, N. Boudet ${ }^{1}$, and L. K. Haikala ${ }^{2}$ \\ ${ }^{1}$ CESR, Observatoire Midi-Pyrénées (CNRS-UPS), Université de Toulouse, BP 4346, 31028 Toulouse Cedex 04, France \\ e-mail: klotz@cesr.fr \\ 2 Observatory, PO Box 14, 00014 University of Helsinki, Finland
}

A\&A 488, 559-564 (2008), DOI: 10.1051/0004-6361:200809952

Key words. ISM: clouds - ISM: individual objects: Cha-MMS1 - ISM: abundances - stars: formation - errata, addenda

Due to a publishing error in our paper (Klotz et al. 2008) Fig. 6 was made of two identical plots. The correct figure is reproduced as Fig. 1 below.
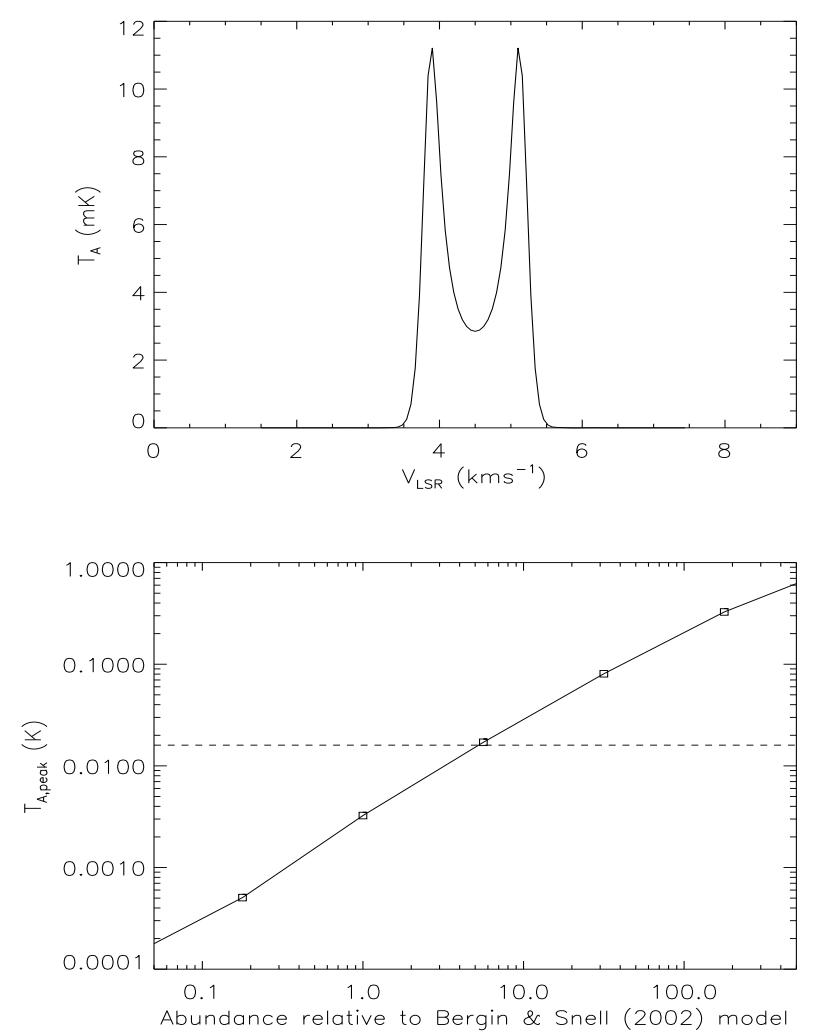

Fig. 1. Top: $\mathrm{H}_{2} \mathrm{O}$ spectrum calculated from the model described in Sect. 5 and in Fig. 5. Bottom: expected peak antenna temperature of the $557 \mathrm{GHz}$ $\mathrm{H}_{2} \mathrm{O}$ line as a function of the scaling factor used to multiply the Bergin \& Snell (2002) abundances. The detection limit (the dashed horisontal line) corresponds to a scaling factor of 5 .

\section{References}

Bergin, E. A., \& Snell, R. L. 2002, ApJ, 581, L105

Klotz, A., Harju, J., Ristorcelli, I., et al. 2008, A\&A, 488, 559 